\title{
U-Lab Cloud: uma proposta de laboratório virtual ubíquo baseado em cloud computing
}

\author{
Rafaela Ribeiro Jardim, UFSM, rjardim@inf.ufsm.br \\ Eduardo Lemos, UFSM, elemos04@gmail.com \\ Fabricio Herpich, UFSM, fabricio.herpich@gmail.com \\ Ricardo Bianchim Gomes, UFSM, ricardo@inf.ufsm.br \\ Roseclea Duarte Medina, UFSM, roseclea.medina@gmail.com
}

\begin{abstract}
Resumo. Este artigo apresenta a proposta do U-Lab Cloud, um laboratório virtual ubíquo baseado em uma infraestutura de Cloud Computing. Esta proposta faz parte do projeto de dissertação de mestrado, divide-se em duas etapas: (etapa I) implantação da plataforma de Cloud Computing, que será apresentada neste trabalho e (etapa II) construção do laboratório virtual (em andamento). Este trabalho apresenta definições, vantagens e desvantagens da utilização de Cloud Computing no ambiente educacional.
\end{abstract}

Palavras- Chave: Cloud Computing, laboratórios virtuais, ubíquo.

\section{U-Lab Cloud: proposal ubiquitous virtual laboratory based in cloud computing}

\begin{abstract}
This paper presents the proposal of U-Lab Cloud, a ubiquitous virtual laboratory based on an infrastructure Cloud Computing. This proposal is part of the project dissertation, is divided into two stages (stage I) implementation of the Cloud Computing platform, which will be presented in this work and (stage II) building the virtual lab (in progress). This paper presents definitions, advantages and disadvantages of the use of Cloud Computing in the educational environment.
\end{abstract}

Keywords: Cloud Computing, virtual laboratory, ubiquitous.

\section{Introdução}

O uso de laboratórios virtuais é essencial para a aquisição de competências práticas (Caminero et al., 2013). Esses laboratórios permitem que o aluno realize simulações e pratique as teorias, sem a necessidade de um laboratório físico, uma vez que a construção dos mesmos exige alto investimento e requer uma infraestrutura adequada. Além disso, outra dificuldade evidenciada é a necessidade de uso exclusivo desses recursos e a rapidez com que os mesmos se tornam obsoletos (Rauen, 2003). Diante deste cenário, surge a necessidade de propiciar um ambiente educacional que forneça recursos computacionais disponíveis de forma dinâmica, simples e com baixo custo.

Desse modo, a infraestutura de Cloud Computing é uma alternativa para a prestação de tais recursos e serviços. Uma das maiores vantagens de utilizar essa solução é o fato de que se pode usufruir das aplicações disponibilizadas em qualquer lugar e independente de qualquer plataforma. Além desse benefício, ao se utilizar Cloud Computing tem-se a possibilidade de reduzir os custos com infraestrutura e licenças de software, essas características tornam essa solução ainda mais atraente. Dentre essas 
vantagens, outro fator que foi considerado para a inserção de Cloud Computing neste trabalho é que a mesma oferece ao usuário muita flexibilidade, já que dispensa a instalação de programas e aplicações. Sendo assim, essa solução pode ser implantada em instituições de ensino que dispõem de computadores com baixa capacidade de processamento, ou seja, os conhecidos "terminais".

Apesar de vários trabalhos abordarem Cloud Computing na educação, tais como (Caminero et al., 2013), (Wang et al., 2010), (Sá et al. 2011) percebe-se uma carência na descrição sistemática do desenvolvimento durante o processo de implantação da plataforma de Cloud Computing, visto que a maioria dos artigos discute técnicas, tipos de serviços, entre outros. Assim, diante da diversidade de plataformas, padrões e sistemas operacionais disponíveis surgiu a necessidade de se estudar e propor uma nova alternativa de infraestrutura para disponibilizar o U-Lab Cloud. Desta forma, evidenciase que a implantação de uma arquitetura Cloud Computing educacional onipresente, escalável e reutilizável para apoiar o compartilhamento de vários tipos de recursos ainda enfrentam grandes desafios.

Contudo, o objetivo deste trabalho é apresentar a proposta do U-Lab Cloud, um laboratório virtual ubíquo, mostrando a viabilidade de implantação de uma nuvem privada, bem como suas analisar suas vantagens e desvantagens.

Este artigo esta estruturado da seguinte maneira: a seção 2 apresenta o referencial teórico; na seção 3 são apresentados os trabalhos relacionados; a seção 4 descreve a metodologia; a seção 5 apresenta a proposta do U-Lab Cloud; já a seção 6 é descrita a análise os resultados. Por fim, são as apresentadas as considerações finais desta proposta de trabalho.

\section{Referencial Teórico}

Nesta seção, são apresentadas algumas definições para Cloud Computing e U-learning, assim como as principais vantagens e desvantagens da adoção desses paradigmas computacionais.

\subsection{Cloud Computing}

A Cloud Computing gera muita discussão na área de Tecnologia da Informação (TI), já que é um conceito novo. Na literatura encontraram-se muitas definições para Cloud Computing. Para Foster et al. (2008), a computação em nuvem pode ser definida como um paradigma de computação distribuída que é impulsionado pelas economias de escala, na qual um conjunto gerenciável de poder computacional, de armazenamento e plataformas consideradas abstratas, virtualizadas e dinamicamente escaláveis são entregues sob demanda para clientes externos através da Internet.

Em Mell e Grance (2011), Cloud Computing é definida como um modelo computacional com a habilidade de permitir, de maneira ubíqua e conveniente o acesso sob demanda a recursos computacionais compartilhados e configuráveis.

Já em Buyya et al. (2008), a computação em nuvem é um tipo de sistema paralelo e distribuído que consiste em uma coleção de computadores interconectados e virtualizados, que são dinamicamente provisionados e apresentados como um ou mais recursos computacionais unificados. Essas definições são importantes, pois caracterizam Cloud Computing e diferenciam de outros paradigmas da computação.

Entre muitas das características de Cloud Computing, segundo Caminero et al. (2013) o uso dessa tecnologia permite o gerenciamento de sistema de computação versátil, já que pode ser implantado, escalado, replicado e atualizado com facilidade em qualquer um dos níveis de computação em nuvem. Para Liang (2011) uma das maiores 
vantagens de utilização dos serviços em nuvem é a preocupação reduzida com a perda de dados ou com a intrusão de vírus. A computação em nuvem é um modelo computacional emergente em que os usuários podem ter acesso ubíquo a suas aplicações a partir de qualquer dispositivo conectado (I.B.M, 2009).

Ao se analisar o modelo computacional em nuvem, percebe-se que um dos seus maiores benefícios é a flexibilidade proporcionada aos seus usuários. Logo, o usuário precisará ter em seu dispositivo/computador apenas de um browser instalado para acessar a aplicação desejada, sem a necessidade de instalação de programas ou mesmo a realização de atualizações.

Outro potencial da Cloud Computing é a possibilidade de compartilhamento dados e de realização de trabalhos colaborativos, práticas que facilitam o trabalho em ambientes educacionais. Por exemplo, ao centralizar o armazenamento de todos os dados em um mesmo lugar e com o mesmo formato, excluí-se a necessidade de conversões e adaptações.

Além disso, ao adotar a Cloud Computing as instituições de ensino não precisam realizar grande investimento financeiro, visto que essa infraestrutura exige pouco recurso computacional, ou seja, pode ser utilizado por com computadores com hardware simples (computadores pessoais). Os gastos com licença de software são suprimidos, pois ao aderir por essa alternativa tem-se a possibilidade de utilizar sistemas operacionais open source (código aberto), gratuitos que podem ser instalados sem restrições ao número de máquinas.

Logo, foi constatado que a utilização desse novo paradigma da computação pode trazer diversos benefícios operacionais e financeiros. Entretanto, existem alguns obstáculos relevantes ao se utilizar computação em nuvem. Como principal desvantagem de utilização desse modelo, Armbrust et al., (2009) aponta a segurança de dados e questiona se é possível manter total segurança no tráfego dos dados. A indisponibilidade de serviço pode ser outro obstáculo para o sucesso desse modelo computacional, dado que é necessário estabelecer conexão com a Internet para acessar os dados.

Existem algumas ferramentas open source disponíveis para a criação de nuvens privadas, como por exemplo, AbiCloud ${ }^{1}$, OpenStack ${ }^{2}$, Eucalyptus ${ }^{3}$, OpenNebula ${ }^{4}$, entre outras. Dentre essas, foram escolhidas duas ferramentas para serem testadas, implantadas e comparadas: Eucalyptus e OpenNebula. Ambas foram escolhidas, pois além de disponibilizarem versões open source, são ferramentas amplamente difundidas no ambiente acadêmico e atendem aos requisitos iniciais deste trabalho.

\subsection{U-learning}

O termo Computação Ubíqua surgiu na década de 90, sendo inicialmente proposto por Mark Weiser. Esse conceito promove a ideia da troca de informações a qualquer hora e em qualquer lugar, por meio da utilização, de forma transparente, inteligente e integrada das tecnologias computacionais (Weiser, 1991). Em Yahya et al., (2010) é mencionada a evolução tecnológica e a ampliação das capacidades de computação e comunicação dos pequenos dispositivos eletrônicos, como responsáveis pelo progresso do electroniclearning (e-learning) para o mobile learning (m-Learning) e do m-Learning para o ubiquitous learning (u-Learning).

Em Despotović et al., (2013), os ambientes de computação ubíqua são definidos como um conjunto de sistemas embarcados (computadores, sensores, interfaces e infraestrutura de serviços), sendo reforçada pelas tecnologias de computação e 
comunicação. Os ambientes computacionais ubíquos permitem aprender a coisa certa, no lugar, no momento certo e no caminho certo (Piovesan, 2011).

Uma característica da Computação Ubíqua (Ubiquitous Computing) é a sensibilidade ao contexto. Em Dey (2001), uma definição para contexto é qualquer informação que possa ser usada para caracterizar a situação de entidades que sejam consideradas relevantes para a interação entre um usuário e uma aplicação. Para Pernas (2009), sensibilidade ao contexto refere-se a tudo que ocorre ao redor do usuário, e que influencia a forma como ele interage com o ambiente e com as outras pessoas. Conforme Dey (2001), a computação sensível ao contexto deve estar ciente do estado do usuário e seus arredores, e deve modificar o seu comportamento com base nesta informação.

\section{Trabalhos Correlatos}

A Cloud Computing propõe a integração de diversos modelos tecnológicos para o provimento de infraestrutura de hardware, plataformas de desenvolvimento e aplicações na forma de serviço (Sá et al., 2011). A virtualização é um dos pré-requisitos para a realização de Cloud Computing (Mell e Grance, 2011). Logo, ela permite a execução de várias máquinas virtuais (VMs) em uma máquina física. Diante das várias vantagens das aplicações de Cloud Computing voltadas para o ensino, são elencadas como as principais: a escalabilidade, o armazenamento centralizado, a flexibilidade, a acessibilidade e os baixos custos (Wenhong et al., 2010).

A Cloud Computing é uma infraestrutura que vem sendo explorada em ambientes educacionais, tais como (Caminero et al., 2013), (Wenhong et al., 2010), (Doelitzscher et al., 2011), (Vouk et al., 2008), (Wang et al., 2010), no entanto, percebese uma carência na descrição sistemática do desenvolvimento durante o processo de implantação das plataformas, sendo que a maioria dos artigos discute funcionalidades, tipos de serviços e de plataformas. Cabe ressaltar, que para a escolha adequada da plataforma de Cloud Computing devem ser considerados alguns aspectos como: a infraestrutura de TI e o tipo de modelo de serviço mais adequado para ser implantado na instituição de ensino. Uma aplicação pode utilizar um ou mais modelos de serviço, dentre eles: Infraestrutura como Serviço (IaaS), Plataforma como Serviço (PaaS) e Software como Serviço (SaaS).

Por exemplo, Wenhong et al., (2010) propõe uma estrutura para gerenciar PaaS em um laboratório virtual baseado em Cloud Computing que implementa o gerenciamento de usuários, recursos e acesso, porém o tema é abordado de forma geral e não é focado nas necessidades de um sistema educacional. Em Doelitzscher et al., (2011) é apresentada uma solução de infraestrutura em nuvem privada contemplando uma combinação de três modelos de serviço (IaaS, SaaS, PaaS), foi desenvolvida na Universidade de Hochschule Furtwangen (HFU). Essa solução permite a criação de máquinas virtuais por meio da escolha de uma imagem e dos pacotes de software selecionados pelo usuário. Da mesma maneira, Vouk et al., (2008) descreve um laboratório virtual, onde foram utilizados serviços de Cloud Computing, desenvolvido pela Universidade Estadual da Carolina do Norte (EUA). Esse laboratório permite que os alunos realizem reservas de VMs de acordo com a sua necessidade, possibilitando a escolha entre imagens básicas ou imagens com aplicações específicas. Atualmente, essa plataforma está sendo utilizada por um grande número de usuários, utiliza o modelo de serviço SaaS e fornece uma variedade de aplicações específicas para diferentes áreas de estudo.

Em Caminero et al., (2013) foi descrito um sistema baseado em Cloud Computing direcionado para o ensino de engenharia. Este sistema, refere-se a um 
laboratório virtual que disponibiliza recursos de hardware e software, onde podem ser desenvolvidos trabalhos práticos a partir de máquinas virtuais (VMs). São mencionados problemas mencionados durante a implantação da plataforma OpenNebula, relacionados a comunicação de rede e a criação das máquinas virtuais (VMs), mas que foram solucionados. Da mesma forma, Wang et al., (2010) cita um laboratório para administração de rede, implementado com a integração das ferramentas Lab Manager e VMware, tendo como característica principal a flexibilidade de recursos.

Em comparação com os trabalhos acima mencionados, o U-Lab Cloud diferencia-se, pois irá proporcionar um ambiente adaptado as especificidades dos alunos, considerando as características individuais, como por exemplo: o perfil cognitivo, o dispositivo utilizado e a velocidade de conexão. Outro diferencial do U-Lab Cloud, é que o mesmo fornecerá aos alunos um ambiente que poderá ser acessado de qualquer dispositivo que disponha de uma conexão de Internet e um browser instalado, facilitando o acesso ao eliminar a necessidade de instalação ou atualização de programas.

\section{Metodologia}

Para o desenvolvimento deste trabalho, denominado U-Lab Cloud, foram elencadas duas etapas distintas. A primeira caracteriza-se pela construção do referencial teórico, onde se buscou estudar os principais conceitos utilizados neste trabalho. Essa primeira etapa, envolve ainda a implantação de duas nuvens privadas, sendo que em uma foi utilizada a plataforma de Cloud Computing Eucalyptus versão 3.4.2. com sistema operacional CentOS e na outra foi implementado o OpenNebula executado no sistema operacional Ubuntu 13.10. Ambas as plataformas foram escolhidas por disponibilizarem versões open source e por apresentarem os requisitos inicias para contexto deste trabalho.

Durante o período de instalação da plataforma de Cloud Computing Eucalyptus, foram testados os hipervisores $\mathrm{KVM}^{5}$ e o $\mathrm{Xen}^{6}$, os dois são suportados pelo Eucalyptus. $\mathrm{O}$ hipervisor, também chamado de monitor de máquina virtual permite que seja o sistema operacional hóspede seja executado, e constrói as interfaces virtuais para as interfaces reais do sistema hospedeiro. A virtualização é o processo de ocultar o hardware físico subjacente de modo que ele possa ser utilizado e compartilhado de forma transparente em vários sistemas operacionais (I.B.M, 2009). O hipervisor Xen, apresentou mais compatível com a plataforma, tornando-se assim o hipervisor utilizado para os testes. Para a plataforma OpenNebula, também foi configurado o hipervisor Xen, por ser compatível com o sistema operacional Ubuntu instalado.

Para a instalação dessas plataformas, foi utilizado o servidor localizado no laboratório do Grupo de Redes e Computação Aplicada (GRECA), na Universidade Federal de Santa Maria. Esse servidor dispõe de um processador Intel Core2 Quad Q9300 $2.66 \mathrm{GHz}$, com 4 núcleos físicos, $8 \mathrm{~GB}$ de memória RAM e 1 disco rígido de 750GB, com sistema operacional CentOS.

Logo, para avaliar o desempenho das plataformas mencionadas, foi efetuado um teste com o auxílio da ferramenta Intel Linpack $^{7}$ versão 11.1.3.006 com intuito de verificar a diferença de performance entre os sistemas operacionais distintos, uma vez que o ambiente OpenNebula foi executado no sistema operacional Ubuntu 13.10 e a plataforma Eucalyptus no sistema operacional CentOS. Essa ferramenta mede a taxa de execução de operações de ponto flutuante da CPU. Os seus resultados são informados em GFLOPS, ou gigaflops, que é a representação da medida em bilhões de operações de 
ponto flutuante por segundo (FLOPS) que o microprocessador do computador consegue manipular.

Para a segunda etapa, em andamento e não contemplada neste artigo, está prevista a construção do U-Lab Cloud, bem como a inserção dos objetos e de atividades relacionadas ao ensino de Redes de Computadores. O desenvolvimento desta etapa envolve a instalação das plataformas Moodle ${ }^{8}$, OpenSim ${ }^{9}$, Sloodle ${ }^{10}$ e WampServer $^{11}$. A escolha do Moodle deu-se pelo fato de ser open source e por ser atualmente o ambiente virtual de aprendizagem (AVA) utilizado nesta universidade. Optou-se pelo OpenSim, por ser uma ferramenta já utilizada em outros trabalhos desenvolvidos nesse grupo de pesquisa e por disponibilizar versões open source. Ele foi utilizado para criar a interface gráfica do U-Lab Cloud e integrado com o AVA Moodle. Logo, o Sloodle foi utilizado para realizar a integração do Moodle com o OpenSim, permitindo assim a inserção de atividades sobre o tema Redes de Computadores, que serão disponibilizadas aos estudantes por meio do U-Lab Cloud. Por fim, foi utilizado o WampServer para criar um servidor local.

\section{Proposta do U-Lab Cloud}

A arquitetura proposta para o U-Lab Cloud, um laboratório virtual ubíquo baseado em Cloud Computing será apresentada a seguir.

Esse ambiente tem por finalidade capturar as informações do contexto do aluno, como por exemplo velocidade de conexão e estilo cognitivo. O estilo cognitivo será identificado após o preenchimento de um questionário com questões relacionadas ao tipo de material que o aluno prefere, como por exemplo texto ou vídeo. Logo, as respostas serão compiladas e a velocidade de conexão será verificada por um framework com a finalidade de criar um perfil para esse usuário. Com o perfil definido, o aluno receberá a apresentação do conteúdo adaptada as suas particularidades.

O U-Lab Cloud fornecerá recursos dinâmicos de hardware e software, possibilitando redimensionar tais recursos conforme a necessidade de uso, visto que será disponibilizado em uma plataforma de Cloud Computing. A Cloud Computing está dividida em cinco camadas: clientes, aplicação, plataforma, infraestrutura e serviço (Webgranth, 2014). Logo, a arquitetura proposta para o U-Lab Cloud abordará o modelo em camadas de serviços, como pode ser visualizado na Figura 1.

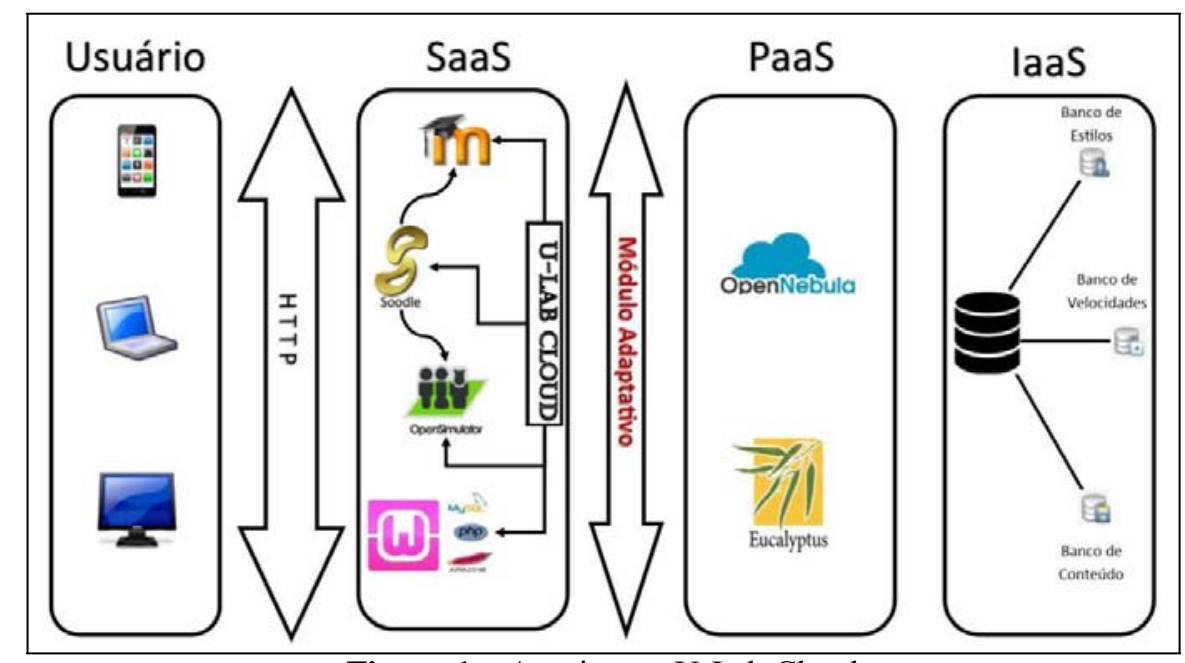

Figura 1 - Arquitetura U-Lab Cloud

Dessa forma, a camada de Aplicações, também chamada de Software como Serviço (SaaS) é a camada mais superficial deste ambiente, fornecerá a interface para 
aluno. Para acessá-la, o aluno precisará ter instalado em seu dispositivo apenas um browser que permita a navegação na Internet. Nessa camada, o aluno terá acesso aos aplicativos disponibilizados, eliminando a necessidade de instalar e executar programas. Ao contrário dos ambientes tradicionais, o U-Lab Cloud dispensará instalação, manutenção ou atualização de programas.

Assim, a instituição não precisará implantar grande infraestrutura para disponibilizar o U-Lab Cloud para seus alunos, e também não precisará de servidores com grandes recursos computacionais. Os alunos terão o U-Lab Cloud rodando na nuvem mesmo que a instituição disponha de computadores com hardware extremamente simples, ao contrário dos ambientes tradicionais.

A camada de Plataformas ou PaaS (Plataforma como Serviço), é a intermediária, facilitará a implantação das aplicações como, por exemplo, o Módulo Adaptativo que realizará o tratamento das informações coletadas sobre o contexto do aluno. O funcionamento desse módulo se dará da seguinte maneira: primeiramente, serão coletadas as informações de preferência informadas pelo aluno ao responder um questionário, logo será verificada a velocidade de conexão do dispositivo que este aluno está utilizando. Após, o Módulo Adaptativo constituirá o perfil do contexto do usuário baseando-se nas informações de velocidade de conexão e preferência de determinados tipos de materiais. Por fim, o Módulo Adaptativo se comunicará com SGBD (Sistema Gerenciador de Banco de Dados) armazenado na camada Infraestrutura ou IaaS (Infraestrutura como Serviço) e adaptará a apresentação do conteúdo no U-Lab Cloud de acordo com a preferência de cada estudante.

O U-Lab Cloud permitirá que os alunos utilizem as versões mais recentes de software, independentemente do hardware que possuam. Caso seja necessário aumentar o número de usuários, tem-se a possibilidade de aumentar esses recursos dinâmicos conforme a demanda, sem preocupação com a infraestrutura física.

\section{Análise dos resultados}

Neste trabalho, foram realizados testes para avaliar o desempenho das plataformas, como mencionado na seção 5. Para realização dos testes, foi utilizada a ferramenta Intel Linpack, com intuito de analisar a diferença entre a performance dos sistemas operacionais instalados, uma vez que o ambiente OpenNebula foi instalado no sistema operacional Ubuntu e a plataforma Eucalyptus no sistema operacional CentOS. Esse teste foi utilizado para verificar o quanto sistemas operacionais distintos impactam na performance das plataformas Cloud Computing.

Com os resultados obtidos com os testes de desempenho, foi possível verificar que ambos os sistemas apresentaram pouca variação de valores. A plataforma OpenNebula foi instalada no sistema operacional Ubuntu 13.10 e atingiu 34,8 GFLOPS, conforme mostra a Figura 2.

Observou-se que a plataforma Eucalyptus executada no sistema operacional CentOS, apresentou um desempenho levemente superior, obtendo 36,1 GFLOPS, como pode ser visualizado na Figura 3. Esses testes foram utilizados para verificar o quanto sistemas operacionais distintos impactam na performance das plataformas Cloud Computing. 


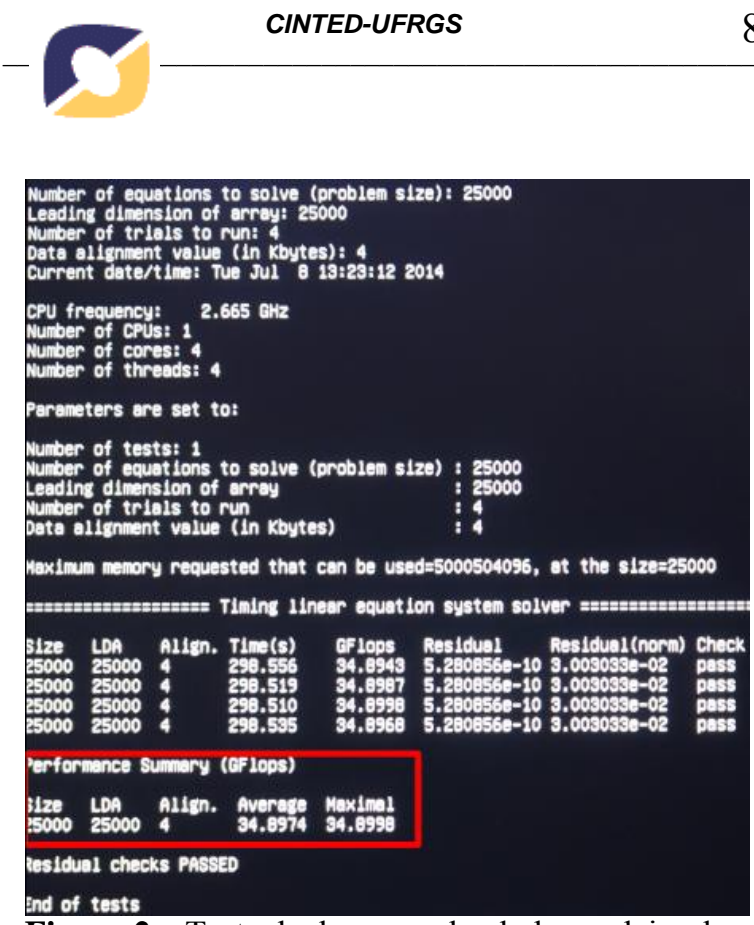

Figura 2 - Teste de desempenho do hospedeiro do OpenNebula, executando no sistema operacional Ubuntu

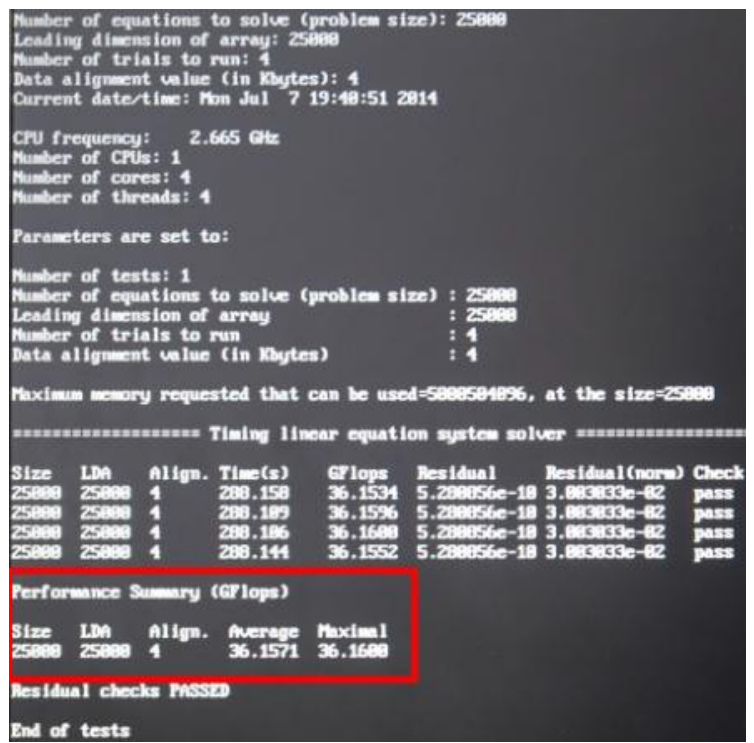

Figura 3 - Teste de desempenho do hospedeiro do Eucalyptus, executado no sistema operacional CentOS

Sendo assim, pode-se afirmar que as variações de desempenho dos sistemas operacionais não impactaram na performance das plataformas de Cloud Computing avaliadas. Essa informação influenciará na definição da plataforma de Cloud Computing que será utilizada para disponibilizar o U-Lab Cloud, considerando também o contexto acadêmico aplicado a este trabalho.

Em relação as funcionalidades, foi possível verificar que ambas as plataformas realizam o gerenciamento de energia, característica importante quando se pretende reduzir os custos com energia elétrica. Na plataforma de Cloud Computing Eucalyptus ocorreu a interrupção das máquinas virtuais ociosas. Essa mesma característica foi encontrada no OpenNebula, sendo realizada pelos sistemas CLUES (Cluster Energy Saving) e pelo UEC por meio do UEC Power Management.

Outra característica encontrada nas duas plataformas foi o balanceamento de carga, ou seja, realizam a divisão das tarefas com o propósito de melhorar o aproveitamento de recursos. Para realizar esse balanceamento a plataforma Eucalyptus emprega o Elastic Load Balancer, ele divide imediatamente o tráfego em trânsito nas aplicações entre os nós do Cluster, por sua vez, o OpenNebula divide os nós da Cloud. Também se observou que as duas possuem um método de conexão bridge e VLAN, caso seja necessário a conexão em diferentes redes.

As duas plataformas de Cloud Computing analisadas disponibilizam interfaces de acesso por meio de SSH (Secure Shell) uma conexão segura entre cliente e servidor ou por meio de uma página web, por HTTP (Hyper Text Transfer Protocol).

Dessa forma, cabe ressaltar que a etapa II encontra-se em processo de construção, onde foram integradas com sucesso as ferramentas Moodle, OpenSim, Sloodle e WampServer. Na figura 4, pode ser visualizado a interface gráfica do U-Lab Cloud no OpenSim. 


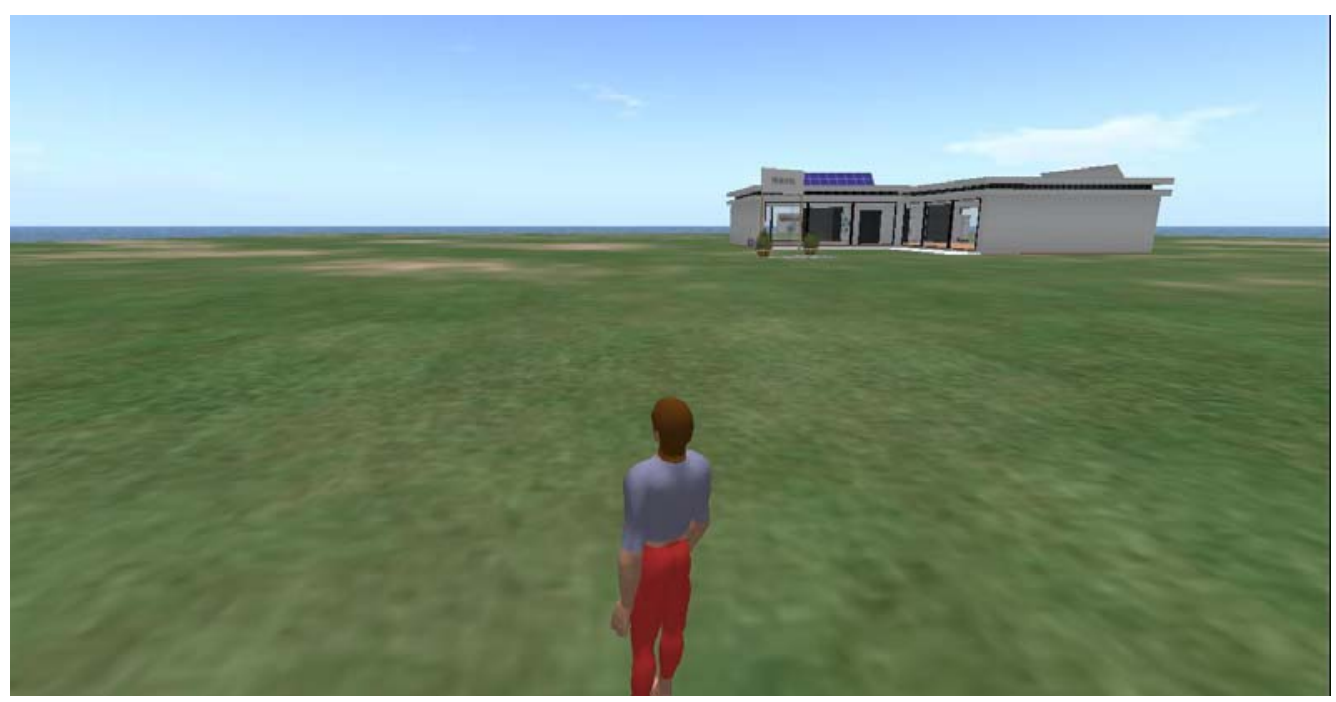

Figura 4 - Desenvolvimento da interface do U-Lab Cloud (em construção)

\section{Considerações finais}

Ao analisar a implementação realizada nesse trabalho, contatou-se que é possível obter um ambiente de computação em nuvem sem maiores investimentos de infraestrutura, em razão de que a mesma dispensa aquisição de novos equipamentos e de equipe técnica.

Este trabalho apresentou a proposta do U-Lab Cloud, um laboratório virtual ubíquo baseado em Cloud Computing, no qual foi realizado uma breve descrição da arquitetura desse ambiente. Assim, o U-Lab Cloud disponibilizará um ambiente com recursos de hardware que poderão ser redimensionados dinamicamente e com características de transparência fornecidas pela Cloud Computing.

Percebe-se que diversos desafios são enfrentados na inserção de Cloud Computing na Educação, entretanto os benefícios desse paradigma superam esses desafios, sendo o maior atrativo dessa solução a economia de recursos financeiros.

Como trabalho futuro, pretende-se analisar a influência ou os possíveis impactos dessa solução no ambiente educacional que será validado no curso de Bacharelado em Ciência da Computação desta instituição.

\footnotetext{
${ }^{1}$ Disponível em: $<$ http://www.ohloh.net/p/abicloud $>$

${ }^{2}$ Disponível em: $<$ https://www.openstack.org/>

${ }^{3}$ Disponível em: $<$ https://www.eucalyptus.com/ $>$

${ }^{4}$ Disponível em: $<$ http://opennebula.org/>

${ }^{5}$ Disponível em: $<$ http://wiki.qemu.org/KVM $>$

${ }^{6}$ Disponível em: <http://www.xenserver.org/>

${ }^{7}$ Disponível em: < https://software.intel.com/pt-br/tags/18403>

${ }^{8}$ Disponível em: $<$ https://moodle.org/>

${ }^{9}$ Disponível em: $<$ http://opensimulator.org/wiki/Main_Page $>$

${ }^{10}$ Disponível em: $<$ https://www.sloodle.org/>

${ }^{11}$ Disponível em: < http://www.wampserver.com/en/>
} 


\section{REFERÊNCIAS BIBLIOGRÁFICAS}

ARMBRUST, M.; FOX A.; GRIFfTTH R.; JOSEPH A.; KATZ R.; KONWINSKI A.; LEE G.; PATTERSON D.; RABKIN A.; STOICA I.; ZAHARIA M. Above the Clouds: A Berkeley View of Cloud Computing. In: Tech. Rep. UCB/EECS-2009-28, EECS Department. University of California, Berkeley, 2009.

BUYYA, R.; YEO, C.; VENUGOPAL, S. Market-Oriented Cloud Computing: Vision, Hype, and Reality for Delivering IT Services as Computing Utilities. In: 10th IEEE international conference on high performance computing and communications, 2008.

CAMINERO, A. C.; HERNANDEZ, R.; ROS, S.; TOBARRA, L.; ROBLES-GOMEZ, A.; SAN CRISTOBAL, E.; TAWFIK, M.; CASTRO, M. Obtaining university practical competences in engineering by means of virtualization and cloud computing technologies. In: Teaching, Assessment and Learning for Engineering (TALE), IEEE International Conference, pp. 301-306, Spain, 2013.

DOELITZSCHER F.; SULISTIO A.; REICH C.; KUIJS H.; WOLF D. Private cloud for collaboration and e-Learning services: From IaaS to SaaS. In: Journal Computing, v.91, pp. 23-42, 2011.

FOSTER, I.; ZHAO Y.; RAICU I.; LU S. Cloud Computing and Grid Computing 360Degree Compared. In: Grid Computing Environments Workshop, University of Chicago, pp.01-10, 2008.

I.B.M. Seeding the Clouds: Key Infrastructure Elements for Cloud Computing Disponível em: <ftp://public.dhe.ibm.com/software/sg/cioleadershipexchange/seeding the cloud.pdf $>$ Acesso em: 05 mai. 2014.

LIANG, X. CRM business cloud computing. In: International Conference On Innovative Computing And Cloud Computing, p. 103-106, New York, 2011.

MELL, P.; GRANCE, T. The Nist Definition of Cloud Computing In: National Institute of Standards and Technology, [S.1], p. 7, 2011.

RAUEN, T. R. S. Uma abordagem alternativa para ensino de redes de computadores. Universidade Federal de Santa Catarina, 2003. 85p. Dissertação de Mestrado.

SÁ, T. T.; SOARES J. M.; GOMES D.G. Cloudreports: Uma ferramenta gráfica para simulação de ambientes computacionais em nuvem baseada no framework Cloudsim. IX Workshop em Clouds e Aplicações - WCGA, Fortaleza, 2011.

VOUK, M.; AVERITT, S.; BUGAEV, M.; KURTH, A.; PEELER, A.; SHAFFER, H.; THOMPSON, J. Powered by VCL - Using Virtual Computing Laboratory (VCL) Technology to Power Cloud Computing. In: Proceedings of the 2nd International Conference on Virtual Computing, pp. 1-10, 2008.

WANG X.; HEMBROFF C.; YEDICA R. Using the VMware vCenter Lab Manager in undergraduate courses in system administration and network security. In: Proc. da 11 ${ }^{\mathrm{a}}$ Conferência ACM de Educação Tecnologia da Informação (SIGITE), Midland, EUA, 2010.

WEBGRANTH. A Complete Reference to Cloud Computing. Disponível em: $<$ http://www.webgranth.com/a-complete-reference-to-cloud-computing $>$ Acesso em: 02 mai. 2014.

WENHONG, T.; SHENG, S.; GUOMING, L. A framework for implementing and managing platform as a service in a virtual cloud computing lab. In: Education Technology and Computer Science (ETCS), Second International Workshop on, pp. 273-276, v. 2, 2010. 